\title{
The evolution of syntax: an exaptationist perspective
}

\author{
W. Tecumseh Fitch* \\ Department of Cognitive Biology, University of Vienna, Vienna, Austria
}

\section{Edited by:}

Constance Scharff, Freie Universitaet Berlin, Germany

Reviewed by:

David W. Dickins, University of Liverpool, UK

Paul M. Nealen, Indiana University of PA, USA

\section{${ }^{*}$ Correspondence:}

W. Tecumseh Fitch, Department of Cognitive Biology, 14 Althanstrasse, Vienna, Austria.

e-mail: tecumseh.fitch@univie.ac.at
The evolution of language required elaboration of a number of independent mechanisms in the hominin lineage, including systems involved in signaling, semantics, and syntax. Two perspectives on the evolution of syntax can be contrasted. The "continuist" perspective seeks the evolutionary roots of complex human syntax in simpler combinatory systems used in animal communication systems, such as iteration and sequencing. The "exaptationist" perspective posits evolutionary change of function, so that systems today used for linguistic communication might previously have served quite different functions in earlier hominids. I argue that abundant biological evidence supports an exaptationist perspective, in general, and that it must be taken seriously when considering language evolution. When applied to syntax, this suggests that core computational components used today in language could have originally served non-linguistic functions such as motor control, non-verbal thought, or spatial reasoning. I outline three specific exaptationist hypotheses for spoken language. These three hypotheses each posit a change of functionality in a precursor circuit, and its transformation into a neural circuit or region specifically involved in language today. Hypothesis 1 suggests that the precursor mechanism for intentional vocal control, specifically direct cortical control over the larynx, was manual motor control subserved by the cortico-spinal tract. The second is that the arcuate fasciculus, which today connects syntactic and lexical regions, had its origin in intracortical connections subserving vocal imitation. The third is that the specialized components of Broca's area, specifically BA 45 , had their origins in non-linguistic motor control, and specifically hierarchical planning of action. I conclude by illustrating the importance of both homology (studied via primates) and convergence (typically analyzed in birds) for testing such evolutionary hypotheses.

Keywords: syntax, cognitive biology, evolution of language, comparative neuroscience, arcuate fasciculus, vocal imitation, human evolution, Broca's area

\section{INTRODUCTION}

\section{NATURALIZING THE LINGUISTIC BRAIN}

Human language, in its mature state, is a complex system that allows us to encode and communicate thoughts and experiences via hierarchically structured signals called sentences. Humans, apparently uniquely among living animals, are born with a capacity to acquire such systems readily and without the need for explicit tutelage: an instinct to learn language. The capacity for syntax is a central component of this system.

The traditional textbook picture of the neural circuitry underlying the human capacity for language involves premotor speech and syntax areas in Broca's region, posterior temporo-parietal areas involved in comprehension, and connections between them involving the arcuate fasciculus. Current opinions about this threecomponent model vary from tacit acceptance in many studies to flat-out rejection by a few (e.g., Lieberman, 2000). Today, it seems clear from modern brain imaging work that parts of this model were right all along (e.g., that Broca's area, especially Brodmann's Area (BA) 44 and BA 45, play an important role in language). Other parts are only partially correct (e.g., brain regions involved in language, especially production, tend to be biased toward the left hemisphere, but earlier scientists erred in seeing this bias as a complete left localization). The "connectionist" (in the Lichtheimian nineteenth century sense, not the twentieth century neural network sense) component of the model has had new life breathed into it by comparative diffusion-tensor imaging (DTI) research (Catani and Mesulam, 2008; Rilling et al., 2008). While the importance of left-lateralization, or the precision with which "Wernicke's area" can be localized, may be questioned, the basic notion that Broca's area and its connections with parietal and temporal cortex play an important role in language, both in speech and in syntax, seems rather clear. In this article I will take this for granted, and inquire into the evolution of these linguistic circuits.

When we inquire into the evolutionary changes characterizing human language, our most solid point of reference will always be differences between human brains and brains of other primates, whether in brain anatomy or in brain function. That is, in evolutionary terms, we are seeking human autapomorphies (traits which differentiate us from our closest relatives, and were not present in the last shared ancestor). Cladistically speaking, the correct comparisons are with our nearest living cousins, the great apes, though both technical and ethical problems with ape neuroscience often force us to rely heavily on more distant primate cousins, especially macaques, to identify evaluate human specializations. So, given the evident absence of language in apes, the fundamental question is "what derived features underlying language arose in the human 
lineage after it separated from our last common ancestor (LCA) with chimpanzees?" This search for differences, for derived traits, is thus a search for human autapomorphies.

However, in asking this question about human specializations we obviously should not ignore the vast amount of neurobiology that we share with other primates, or other vertebrates more generally. Modern neuroscience is full of neural synapomorphies (traits we share with relatives, by common descent). With other vertebrates we share virtually all aspects of neurotransmitter chemistry, neuronal morphology, and brainstem circuitry, along with many detailed aspects of neural processing (e.g., the circuitry of the retina or superior colliculus, or the layout of motor circuitry in the cerebellum or basal ganglia). With other mammals we share the possession of a six-layered neocortex and the strongly conserved pattern of connectivity between its layers. When it comes to chimpanzees, it is no exaggeration to say that virtually all known aspects of neuroanatomy appear to be shared, aside from gross size differences, and there are actually few known differences between apes and other Old World primates (for an exception see Nimchinsky et al., 1999). So whatever the neural differences underlying the human capacity for language are, they must always be viewed in the context of an ocean of similarity. Human neural autapomorphies are needles in a synapomorphic haystack.

From a biologist's viewpoint, there is of course nothing special about being "special." Every living species possesses autapomorphies of some sort (otherwise it would not be recognizable as a species), and these specialities always are understood in the context of a much larger set of synapomorphies. We should be no more surprised by human neural autapomorphies tied to language than we are to find that star-nosed moles have a highly specialized somatosensory cortex for their nasal "fingers" (Catania, 1999), or that echolocating bats have specialized auditory regions for their echolocation calls (Suga et al., 1987). It is thus unfortunate that when considering our own specializations, humans have a tendency to invest them with especial significance. This is a mistake. Biologists and neuroscientists should not allow our investigation of neural autapomorphies to be conflated with philosophers' longrunning search for defining features of humanity, features that, as a modern replacement to the uniquely human soul, might be misused to justify moral or ethical stances. An ethics built upon empirically determined autapomorphies is built upon shifting sands, as scientific findings might demand change at any point. Thus it is important to divest our investigations of any neural differences that exist from larger, and essentially philosophical, questions about why humans are "special" in ethical or moral senses.

Our task, as neurobiologists, is simply to identify the various neural mechanisms underlying language and, using the comparative approach, to determine which are synapomorphic and which are not. And, given that language relies on widely shared neural foundations, we should not be surprised to find abundant synapomorphies, nor should we take such findings as evidence against the existence of autapomorphies. My goal in this paper is to explore the evolution of human neural autapomorphies involved in language, with a focus on syntax. This exploration will adopt a fully comparative approach, using what is known from research on non-human primates, mammals more generally, and birds, to contextualize whatever few autapomorphies humans possess in terms of the much wider foundation of shared synapomorphies.

Furthermore, I will attempt to clarify and defend one particular approach to the evolution of novelties, based on the idea of exaptation: that complex structures, evolved in one functional context, can change their function, and be put to work in new domains, often carrying with them traces or constraints due to their prior function. This exaptationist perspective is in full accordance with the truism that evolution has no foresight, or with the evocative image of evolution as a "tinkerer," that gets by with whatever parts or raw materials happen to be available (Jacob, 1977, 1982). Nonetheless, the notion of exaptation when applied to human cognitive evolution has evoked skepticism or even hostility (e.g., Dennett, 1995; Buss et al., 1998; Newmeyer, 1998; Pinker and Jackendoff, 2005), even by those who accept the more general notion of evolution as tinkerer. Thus I will start with a brief exploration of the general concept of change of function (which was a cornerstone of Darwin's model of evolution) and its resurrection in the term "exaptation" by Gould and Vrba, and then very briefly outline some of the objections that have been made to this concept, and the closely related concept of "spandrels," as applied to human cognition and language.

Next, I will outline in detail three exaptive hypothesis concerning human language, which illustrate how an explicitly exaptive model, built on a firm comparative foundation, can both make sense of human specializations and provide further testable predictions for neuroscientists "on the front line" of comparative and functional neuroscience. These hypotheses are, for the most part, built upon the ideas of other scholars, though they are not always framed in the context of exaptation. Although I find each of these hypotheses plausible, my goal here is not to convince anyone that they are true. Rather, my purpose is to demonstrate that exaptive hypotheses about language evolution are plausible, and are part and parcel of a standard biological approach. Further, I detail some testable predictions in the hope that some future neuroscientists will attempt to test such models. In the final part of the paper, I will briefly describe the virtues of a broad comparative approach, incorporating research on both mammals and primates (to discern homologies and ancestral states) and birds (to exploit the hypothesis-testing power of convergent evolution). I believe that there is real value to be gained for evolutionary neuroscience in adopting the rigorous and explicit comparative approach that evolutionary biologists have perfected in the last few decades (complete with the admittedly somewhat ungainly terminology of synapomorphies and autapomorphies).

Naturalizing the human brain demands that we acknowledge both its widely shared foundations with other animals, and recognize at the same time its unique specializations. Our understanding of ourselves will be incomplete without a clear-eyed acceptance of both classes of traits. My goal in this paper will be to investigate how autapomorphies characteristic of our species, and specifically involved in language, might have evolved. More specifically, I aim to defend the proposition that change in function, one of Darwin's favorite evolutionary phenomena, might have played an important role in language evolution and particularly for syntax. From this "exaptationist" perspective, I will argue with many others that precursors for some language functions will be found not in 
primate communication, but in other aspects of behavior such as motor control or thought. This is by no means a new suggestion, but I will attempt to clarify this exaptive perspective by offering three quite specific models of possible exaptive phenomena, where a circuit built for one function is put to use in another, that are tied to human language.

\section{EXAPTATION AND CHANGE OF FUNCTION}

Darwin was famously a gradualist, suggesting that his entire theory would fall apart if one example of evolutionary leaps, or saltations, could be shown. Gradualism, at its simplest, is the assumption that the distribution of phenotypes across evolutionary time is smooth and continuous (like body mass or height) rather than discrete and discontinuous (like eye or flower color). This is a commitment to a continuum of phenotypes. Darwin's gradualist perspective was weakened considerably in the modern evolutionary synthesis of genetics and evolutionary theory, initially by the fact that genes are, by their nature, discrete. More recently, the assumption of a smooth relation between genotypic and phenotypic change has been challenged by the discovery of homeotic "master control genes" like HOX or PAX genes in which small genetic changes have drastic phenotypic effects. Nonetheless, a smooth continuum of phenotypes appears to be common in many traits, and such smoothness is assumed to be operative by many models of selection (e.g., the Price equation). So gradualism survives, but in a weaker form than Darwin considered to necessary.

A different kind of potential "smoothness" in evolution involves continuity of function. In this case, Darwin was a discontinuist, for he gave multiple examples of "changes in function," and he saw these as exemplifying a major driving force in the evolution of complexity. For example, in discussing the evolution of vertebrate lungs, Darwin proposed that these structures first evolved as complex swim bladders involved in buoyancy control in ancestral fish. As our aquatic ancestors became air breathers, the function of these ancestral swim bladders was converted to that of lungs, which function in the respiratory exchange of gases. Darwin cited several other examples of change in function, including the separate use of the swim-bladder in hearing in some fish, and from flowers and barnacles. Although Darwin gave no specific term to "nascent" traits in which function changed, later authors termed the precursor versions "pre-adaptations." More recently (Gould and Vrba, 1982) objected to the term "pre-adaptation" because of its teleological connotations of evolutionary foresight, and coined the term "exaptation" for the end-product of an evolutionary change in function. Gould and Vrba argued convincingly that such changes in function are ubiquitous in evolution, so much that most complex traits have gone through at least one change in function.

Darwin recognized the exaptive process of evolutionary change of function, combined with gradualism, as a crucial factor in the evolution of complex organs for a simple reason: it is often difficult to see how a small rudiment of an organ could have any adaptive function until it exceeded a certain size or complexity. What good is a tiny lung, that could only fulfill a small proportion of the individual's respiratory needs? What good is a tiny wing, that could never allow an organism to become airborne (Kingsolver and Koehl, 1985)? By the assumption of continuity of function, these are big problems. But as soon as we consider the possibility of change of function, the answer becomes obvious: lungs started out as swim bladders (where a little buoyancy might still be useful) and wings as temperature regulators (where a small radiating or insulating surface might make a considerable contribution). For further examples of change of function (see Gould and Vrba, 1982), and for a critique and clarification of some of the conceptual issues this raises (see Reeve and Sherman, 1993; Dennett, 1995). It should be noted that it appears that Darwin was incorrect in his swim-bladder example about the direction of change. It now appears that lungs evolved first (in early fish, who also possessed gills) and were later converted to complex swim bladders in later fish radiations [see Fitch (2010) for this and other examples of exaptations related to speech].

\section{EXAPTATION IN COGNITIVE EVOLUTION}

Given the established fact that organs can change their function, and the importance of this fact for evolutionary theory, it is somewhat surprising that exaptation has been dismissed (or worse, ignored) by many researchers interested in human cognitive evolution (cf. Gould, 1991; Buss et al., 1998). This is particularly true in language evolution, where a continuity of a function in communication is assumed by many (e.g., Pinker and Bloom, 1990; Pinker and Jackendoff, 2005) while changes in function are seen by others as an explanatory necessity (e.g., Berwick, 1997; Chomsky, 2010).

Part of this debate can be readily resolved by adopting a multi-component perspective, wherein human language capabilities enlist several interacting but independent mechanisms. Many of these mechanisms will be shared with other animals, but several appear to have changed considerably since the LCA of humans and chimpanzees (hereafter, the LCA), including those involved in signaling, syntax, and semantics (cf. Fitch, 2010). From a multi-component perspective it is perfectly plausible that some novel aspects of language (e.g., speech production or hearing) are fully continuous in function from the LCA, whereas others may constitute exaptations where function has changed. Prominent candidates for exaptive change are the computational mechanisms subserving linguistic syntax, the topic of the current article. But to illustrate the point I will start with a less controversial and better understood example, drawn from the neural circuitry underlying language. First it is necessary to clarify how a multicomponent perspective on contemporary language circuits leads to implications for a multi-stage model of language evolution.

\section{EXAPTATION AND DIFFERING MODELS OF PROTOLANGUAGE}

A logical concomitant of a multi-component perspective on language evolution is a notion of intermediate stages during human evolution in which certain components of language were present, while others were either lacking entirely or not integrated into a linguistic system. For those novel aspects of language that evolved since the LCA, the term "protolanguage" is often used to denote such a precursor system, an evolutionary way-station on the path to full modern language. The term "protolanguage" was first used in an evolutionary context by anthropologist Gordon Hewes (1973), who introduced it in the context of a gestural protolanguage hypothesis, by which early stages of linguistic signaling were in the manual/visual domain. It was further popularized by linguist 
Derek Bickerton, who used the term to denote a lexical model of protolanguage, in which spoken words formed the first stage of language evolution (Bickerton, 1990, 2000, 2007). One of the oldest models of protolanguage dates back to Darwin (1871), who suggested that the earliest stages of human language were musical, with no semantic content. This notion of "musical protolanguage" or "prosodic protolanguage" has more recently been embraced by a number of scholars (Richman, 1993; Brown, 2000; Mithen, 2005; Fitch, 2006). For more detail on these and other conceptions of protolanguage (see Fitch, 2010).

Different models of protolanguage constitute different hypotheses about the nature of the mechanisms involved in modern language, along with an implied order in which these mechanisms were acquired during human evolution. Often, these models also offer, whether explicitly or implicitly, hypotheses about the function(s) subserved by different mechanisms as well. For example, Bickerton (2010) suggests that the first words served the communicative function of displaced reference (allowing early hominins to communicate about distant food sources), and that this putative function has remained continuous since that time. In contrast, Darwin's model suggests that the initial function of complex, learned signals was courtship and territorial defense, in direct analogy to birdsong. Darwin's musical protolanguage model thus entails a change of function, from non-verbal communication to semantic communication of propositional knowledge, during hominin evolution. Bickerton's model is thus continuist in this respect, while Darwin's is exaptive, involving a change in function.

Peter MacNeilage, a speech scientist, has offered an exaptive hypothesis for the evolution of speech, where the oscillatory lip, jaw, and tongue movements involved in producing consonant/vowel alternations in human speech we co-opted from the basic mammalian circuitry controlling chewing, licking, and suckling (MacNeilage, 1998). Like Darwin's swim bladder example, MacNeilage's hypothesis suggests that the complex and coordinated motor activities used in speech were taken over from the already complex and coordinated motor control that had evolved, over tens of millions of years, in ancestral mammals to subserve feeding. In the commentary following this article, many critics suggested that a continuity of function was more likely (and thus that human speech evolved from primate vocalizations), but none of them questioned the general plausibility of MacNeilage's exaptive explanation.

Another prominent exaptive model for language evolution is due to Noam Chomsky (1968, 1976, 2010), who argued that the roots of the generative capacity of language should be sought in private conceptual abilities, rather than in communication of knowledge between individuals. His argument, echoed by various prominent biologists like Jacques Monod and Salvador Luria, is that language is far more powerful than it needs to be for communication. Thus, the vast reach of language into all aspects of human cognition cannot be explained by a step-by-step expansion of communication abilities, because most of the sentences we can generate and understand would have no conceivable selective advantage (cf. Piattelli-Palmarini, 1994; Berwick, 1997). In contrast, a flexible and extendable system of thought would always be of considerable adaptive value, because even small advantages in thought can prove valuable if they allow an individual to out-think conspecifics (cf. Jolly, 1966; Humphrey, 1976; Byrne, 1997). In other words, language appears to be more powerful than needed for everyday communication but appropriately powerful as a medium for creative, generative thought. Again, this is simply an exaptive hypothesis like many others, where the function of one aspect of language has changed, over evolutionary time, from conceptual to communicative, but this particular exaptive hypothesis has been singled out for criticism by many authors (Pinker and Bloom, 1990; Newmeyer, 1998; Jackendoff and Pinker, 2005; Pinker and Jackendoff, 2005), so much so that linguist Frederick Newmeyer confessed to finding Chomsky's ideas on this front "utterly implausible" (Newmeyer, 1998).

As this brief review illustrates, there is considerable disagreement about the importance of exaptive change in function in the evolution of human language, and opinions are particularly polarized in the domain of syntax. Of course, one's model of language evolution depends on one's conception of contemporary language, and some real disagreements stem from such underlying theoretical biases. On the other hand, the many examples of change of function in other evolutionary domains force us to consider exaptive hypotheses concerning language with an open mind. The conversion between swim bladders and lungs, or between radiators and insect wings, may not appear intuitively plausible, but both are taken very seriously by contemporary evolutionary biologists.

Furthermore, when continuity of function is simply assumed without argument, this assumption may conceal further problematic assumptions. For example, Clark (2011) shows how Bickerton's model of language evolution, based on continuity of communicative function, makes several problematic implicit assumptions that Bickerton himself fails to consider. Furthermore, the assumption of functional continuity may block consideration of alternative hypotheses. For example, it was assumed for many years that the only plausible explanation for the descent of the human larynx was for speech communication (Lieberman, 1968, 1975, 1984). This led researchers to overlook examples of laryngeal descent in multiple animal species (e.g., Fitch and Reby, 2001; Weissengruber et al., 2002; Frey and Riede, 2003), all of which lack speech, and which clearly demonstrate that a descended larynx can serve adaptive functions other than spoken language (see below for the importance of convergent evolution in such inferences).

Thus, rather than formulating the problem of language evolution as the transition from non-human primates to modern human language, in all its glory, we should rather investigate models in which several stages of protolanguage existed. It is less important to know when these different stages occurred (e.g., in Australopithecus or Homo erectus) than it is to construct plausible scenarios in which specific novel abilities evolved, building on pre-existing mechanisms but extending them in the required direction. For example, we know that humans evolved novel speech control mechanisms, so one stage in human evolution must certainly have involved this capacity. However, many organisms have vocal imitation but lack language, so we must further posit additional evolutionary events in which syntactic or semantic abilities evolved. It is of course possible that multiple capacities evolved simultaneously, but to me it seems much more likely that each of these capacities evolved at different times, involved different circuits, and possibly served different adaptive functions. It is equally 
possible that some of these stages were exaptive (building on noncommunicative precursors) while others were characterized by continuity of function.

\section{THREE EXAPTIVE HYPOTHESES IN THE EVOLUTION OF LANGUAGE}

In the rest of this article I will consider the evolution of the various mechanisms underlying modern linguistic abilities, taking seriously the possibility of one or more changes in function.

\section{EXAPTIVE HYPOTHESIS 1: SPEECH MOTOR CONTROL AND DIRECT CORTICO-AMBIGUAL CONNECTIONS}

While humans are gifted vocal imitators, picking up words and melodies in their environment spontaneously, other primates are not: a chimpanzee raised in a human home will never learn to speak or sing (Yerkes and Yerkes, 1929; Hayes, 1951). This is not due to their peripheral vocal anatomy: most mammals can dynamically lower their larynx to a position that would allow different vowels to be produced (Fitch, 2002, 2010). Furthermore, vocal tract deficiencies would not account for the failure of chimpanzees to imitate melodies, which would require only laryngeal control. Thus, the biological explanation of the lack of complex vocal learning in non-human primates must have something to do with brain structure.

A prominent hypothesis for what, precisely, accounts for the difference between humans and other primates was developed by Uwe Jürgens and his colleagues, on the basis of initial observations by the comparative neuroanatomist Hans Kuypers, and I thus call it the "Kuypers/Jürgens direct connections hypothesis" (Kuypers, 1958a,b, 1973; Jürgens et al., 1982). Kuypers explored the connections from motor cortex to subcortical motor systems in the brainstem and spinal cord, and compared these between cats, nonhuman primates (chimpanzees and macaques), and humans. He used Nauta/Gygax staining to document axonal degeneration after lesions (in animals) or strokes (in humans) to the motor cortex. He examined in particular the motor neurons controlling laryngeal muscles, located in the nucleus ambiguus of the medulla. He found that there were projections from cortical motor neurons directly onto these motor neurons only in humans. In cats or nonhuman primates, only multi-synaptic indirect connections were present to ambigual motor neurons. Interestingly, Kuypers found that chimpanzees and macaques do have direct connections to brainstem nuclei controlling the face (lips and jaw), while cats lack these.

This argument is consistent with lesion data: while lesions to motor cortex can induce long-lasting mutism in humans, matched lesions have no effect upon vocal production in monkeys (Sutton et al., 1974; Aitken, 1981; Jürgens et al., 1982). Lesions to lateral cortex often severely disrupt human speech, but spare innate species-typical vocalizations like laughter and crying (Foerster, 1936; Groswasser et al., 1988). Finally, since non-human primates have direct cortico-motor connections to the jaws, tongue, and lips, the frequent use by chimpanzees of learned, un-phonated "vocalizations" like jaw clacks, lip smacks, and lip buzzes ("raspberries") in volitional, goal-directed communication (Marshall et al., 1999; Reynolds Losin et al., 2008) is consistent with the general idea that direct connections are needed for learned, volitional communication. All that is missing in chimpanzees is laryngeal control. These data, and other convergent data from birds (see below), has led many comparative neuroscientists to endorse the idea that direct cortico-motor connections to the larynx play a key role in human speech abilities (e.g., Myers, 1976; Deacon, 1992; Holstege and Ehling, 1996; Striedter, 2004).

How did humans develop direct connections that are lacking in other primates? In this case we have a very clear candidate for a mammalian "pre-adaptation" for this human situation: the cortico-spinal tract. The cortico-spinal tract is a major descending pathway from motor cortex down to motor neurons within the spinal cord. Cortico-spinal axons originate in pyramidal neurons in layer $\mathrm{V}$ of the neocortex, mostly in primary motor cortex but also from premotor cortex, the supplementary motor area (SMA), cingulate gyrus, and somatosensory cortex. A clear homolog of the cortico-spinal tract is present in all mammals. A closely related set of cortico-motor projections make up the cortico-bulbar tract, which project from cortex down to various brainstem motor nuclei found in the trigeminal (CN V, controlling the jaw), facial (CN VII: lips and other facial musculature), and hypoglossal (CN XII, controlling the tongue) nuclei. Thus, in primates, the cortico-bulbar and cortico-spinal tracts together innervate motor neurons above and below the key laryngeal motor neurons located in the nucleus ambiguus ( $\mathrm{CN} \mathrm{X}$, containing the motor neurons of the vagus nerve complex, including the superior and recurrent laryngeal nerves). I suggest that either, or both, of these tracts provided a precursor of the direct cortico-ambigual connections found in humans. Specifically, I propose that the developmental processes underlying axonal growth and synapse-formation in the cortico-spinal tract were "exapted" to generate cortico-ambigual connections found in adult humans and which play a key role in speech.

Why do such direct connections develop in humans but not other primates? An intriguing hypothesis has been put forward by Terrence Deacon, involving competition between an innate call system based in the brainstem, and the cortico-motor system. Most mammals share a basic system for producing species-typical calls, involving a "control center" in the periaqueductal gray (PAG) of the midbrain (Jürgens, 1994; Holstege and Ehling, 1996). This system can produce the entire species-typical vocal repertoire of cats or squirrel monkeys, or innate vocalizations like laughter and crying in humans, and does not rely upon cortical input (hence, anencephalic human babies who lack a neocortex can still smile, laugh, and cry normally). Deacon has suggested that, during development, there is competition between this prepotent brainstem system and cortical axons projecting down through the brainstem, which transiently connect to laryngeal motor neurons in the nucleus ambiguus, but are out-competed by the innate vocal system projections from PAG and other centers (Deacon, 1992, 1997). By Deacon's hypothesis, evolving the kind of direct cortical control over vocalizations typical of humans would require a reduction in the innate call system, and perhaps also the innate call repertoire. This hypothesis has various testable predictions, and even suggests that direct cortico-motor connections could be elicited experimentally in other mammals, if the innate call system was reduced by lesions at the right stage of development (along the lines of Roe et al., 1992). 
Obviously, speech motor control is closely tied to the auditory/vocal modality of communication, and speech is not synonymous with, or even necessary for, human language. However, this aspect of speech does provide a simple, clear example of how a particular neural circuit involved in spoken language might have evolved via exaptation of a pre-existing motor circuit found in other primates. While the Kuypers/Jürgens hypothesis is the best available explanation of human vocal control capacities at present, the exaptive hypothesis sketched above remains speculative at present. However, it is clearly both testable, and consistent with a considerable body of knowledge from comparative neuroscience.

\section{EXAPTIVE HYPOTHESIS 2: INTRACORTICAL CONNECTIONS INVOLVED IN SPEECH}

While the previous example focused on speech motor output, another well-known example of an important circuit involved in spoken language is provided by the arcuate fasciculus connecting temporal/parietal regions to motor and premotor cortex. This white-matter fiber tract was originally discussed in Wernicke's analysis of "conduction aphasia” (Wernicke, 1874), but its importance was highlighted in some classic papers by Norman Geschwind $(1965,1970)$. Wernicke's original argument concerned the necessity, in speech, of an exchange of information between auditory areas which store the sound of a word and motor areas which mediate the motor outputs capable of generating that sound with the articulators. This idea was (over)extended by Lichtheim and Geschwind to include a third hypothetical association area in the angular gyrus. But Wernicke's basic notion that the ability to learn to vocally produce heard sounds (such as words or melodies) requires connections between auditory and motor centers is sound, and has withstood the test of time. For a detailed history of this discussion (see Catani and Mesulam, 2008).

The advent of DTI and in vivo tractography has breathed considerable new life into these old ideas, documenting differences between human brains and those of other primates (Rilling et al., 2008). As in the previous example, there appear to be significant differences among species in the intracortical white-matter connections between temporal, parietal, and frontal regions that are plausibly related to speech motor control (see Petkov and Jarvis, this issue). In particular, Rilling and colleagues found that while the macaque homolog of the arcuate fasciculus makes only specific and limited connections between the superior temporal gyrus and regions of the lateral prefrontal cortex, the human arcuate makes rich and extensive connections to the middle and inferior temporal gyri as well. This expansion of the connectivity from prefrontal regions to essentially the entire temporal lobe may be linked to a relative expansion in humans of both frontal (Deacon, 1997; Schoenemann et al., 2005) and temporal regions (Rilling and Seligman, 2002). This expanded connectivity was also found, but to a limited degree, in one of the four chimpanzee brains scanned.

The expansion and elaboration of the arcuate fasciculus in humans has a more interesting implication in the context of the current topic of exaptation, because many have noted that this pathway is involved not only in speech imitation but also in some aspects of syntax processing. Although Broca himself viewed his eponymous area as primarily involved in speech output, it has become abundantly clear that premotor areas such as BA 44 are also involved in auditory comprehension, and syntax processing in particular (e.g., Caramazza and Zurif, 1976; Grossman, 1980; Friederici et al., 2000). Because this issue is more thoroughly reviewed elsewhere in this issue, I will not go into the details here.

My main point is to suggest that these two functions - auditory/motor exchange and syntax production and comprehension - are not unrelated. Specifically, I hypothesize that reciprocal auditory/motor connections that initially evolved in the context of vocal imitation (an output function) could later be exapted for use in syntax comprehension (an input function). The logic underlying this suggestion is similar to that underlying the motor theory of speech production (Liberman et al., 1967; Liberman and Mattingly, 1985; Galantucci et al., 2006; Feenders et al., 2008; Pulvermüller and Fadiga, 2010) or more recent ideas about the role of the mirror neuron system in linking motor action and visual perception in the support of social cognition or empathy (di Pellegrino et al., 1992; Arbib, 2005; Iacoboni et al., 2005; Keysers and Gazzola, 2006). The prefrontal component of this system is, at birth, concerned basically with motor control. It develops, through babbling and later practice with speech production, a quite complex repertoire of automatized vocal actions. This initially occurs with auditory guidance, but has little significance for auditory comprehension itself. However, once such a learned cortical repertoire exists, it could provide a useful, highly articulated basis for auditory phonetic comprehension (knowing what a sound is based on the actions that would be needed to produce it) and, at a higher level, syntactic, or semantic (knowing what a sentence means based on the structures that would be needed to generate that meaning).

As phrased above, this hypothesis could apply either to ontogeny (during individual brain development) or phylogeny (during the evolution of the required neural circuits in the species). However, one reason to doubt a purely ontogenetic interpretation of syntactic circuitry is provided by studies of signed language (Bellugi et al., 1990). Although the output modality of sign is obviously different from that of speech (involving mostly limb movements, and monitored via vision and somatosensory systems), the syntactic processing of sign nonetheless relies on a brain region identical to, or at least greatly overlapping, Broca's area in spoken language (Horwitz et al., 2003). This suggests that the appropriate connections develop reliably in all humans, regardless of linguistic modality, and thus suggests an influence of an evolved pattern of connectivity in addition to the role of cortical plasticity during individual development.

Exaptive hypothesis 2 thus suggests that the intracortical connections of the human arcuate fasciculus initially evolved for the specific purpose of vocal imitation. These connections, once in place, were then again exapted for use in the more complex task of syntactic comprehension, and particularly between premotor control regions and posterior regions involved in semantic interpretation. This exaptation would have constituted a second evolutionary event, occurring afterward and perhaps for different selective reasons from the first. 


\section{EXAPTIVE HYPOTHESIS 3: THE EVOLUTION OF BROCA'S AREA (BA 44 AND 45)}

The third exaptive hypothesis considered here is closely related to the previous one, and concerns the evolution of specific cortical regions involved in syntax. While the previous hypothesis is hodological, concerned with connections, this one is cytoarchitectonic, and concerned with the computational specializations of specific cortical regions. This hypothesis builds largely upon the work of Angela Friederici and her colleagues (Friederici et al., 2006; Anwander et al., 2007; Bahlmann et al., 2008). It is widely recognized that the left inferior frontal gyrus (LIFG), the traditional site of "Broca's area," is subdivided into multiple regions distinguishable on the basis of cytoarchitecture, receptor distributions, and connectivity (Amunts et al., 1999; Zilles, 2004). Four core regions can be distinguished, the first three designated by their BA:

1. The inferior portion BA 6 - premotor and supplementary cortex;

2. BA 44 - "pars opercularis" - just anterior to BA6;

3. BA 45 - "pars triangularis" - anterior to BA 44;

4. "Deep frontal operculum"-inferior to BA 44, and curling under the temporal lobe.

BA 44 and 45 are the core of Broca's area, as classically defined. In an important study, Horwitz et al. (2003) investigated brain activation during signing and speech by fluent bilinguals, and then correlated the brain activation patterns with cytoarchitectonic maps. They found that BA 44 was activated not only during speech or sign production, but also during complex, volitional movements of the limbs or vocal apparatus with no linguistic content. In contrast, BA 45 was activated only during the production of either spoken or signed language (complex narratives, so including phonological, syntactic, and semantic components). In both cases, activations were significantly above baseline only in the left hemisphere. These data suggest that Broca's region is parcellated into regions that differ in the degree to which they are tied to motor control.

The idea that Broca's area is made up of computationally distinct regions receives strong support from neuroanatomical research on both humans and monkeys (Rempel-Clower and Barbas, 2000; Anwander et al., 2007; Gerbella et al., 2010). Cytoarchitectonically, each of the four regions above is distinct in terms of the granularity of layer 4 of neocortex. BA 45 has a well-developed granular layer 4 (it is "granular"), while layer 4 is present but poorly developed in BA 44 (which is thus termed "dysgranular"). The deep frontal operculum and BA 6 are "agranular": they lack any distinct layer 4 (a trait typical of motor regions).

Connectivity also varies among these regions. Tracer studies in macaques, which remain the "gold standard" for analysis of connectivity, clearly show that differences in granularity reflect fundamental differences in connectivity (Rempel-Clower and Barbas, 2000), and such studies also show that BA 45 in macaques clearly divides into two distinct components. BA 45B is heavily connected to eye movement circuitry such as the frontal and supplementary eye fields. While BA 45A also makes extensive frontal connections, it is unique in having strong connections to superior temporal and auditory areas (Gerbella et al., 2010) and seems more directly homologous to human BA 45 . Human tractography, using DTI and related methods, also reveals a clear parcellation of Broca's area (Anwander et al., 2007), with BA 44, BA 45, and the deep frontal operculum having distinct patterns of connectivity.

There are two distinct exaptive hypotheses about the evolutionary origins of language-specific functions in Broca's region, and particularly BA 45. The first, which I will dub the "premotor origins hypothesis," continues a long line of suggestions that the underlying computations of human language syntax are related in some way to motor control and motor planning. Many theorists have suggested that the hierarchical nature of linguistic structures is related in some way to the hierarchical nature of motor planning (e.g., Lashley, 1951; Lieberman, 1984; Kimura, 1993; Allott, 1994; MacNeilage, 1998). This suggestion leads to a more concrete suggestion in the current context: that the premotor functions of BA 6 and the deep frontal operculum (with their agranular layering pattern) provided the precursor of computations more directly characteristic of language through a gradual "granularization" of gray matter, and strengthening of pre-existing connections to other regions of cortex (e.g., via the arcuate fasciculus to parietal and temporal regions). Starting with BA 44, and progressing to BA 45 , this would lead to a broader and more abstract computational role for Broca's area, culminating in the amodal, granular BA 45.

A second (and not mutually exclusive) hypothesis has not, to my knowledge, been previously suggested. Apes and Old World primates like chimpanzees and macaques have binocular vision and trichromacy, reflecting an increased importance of the visual modality relative to sound and olfaction. This is reflected specifically in a heightened awareness of the gaze of others, and volitional control of one's own eyes, both of which play important roles in Old World primate social behavior and understanding (Scaife and Bruner, 1975; Emery et al., 1997; Emery, 2000). For review of the importance of gaze and visual cueing in the evolution of cognition and language see (Fitch et al., 2010). While the movement of the eyes is clearly a motor function, its control requires strong intracortical communication from visual and multi-modal areas. When such visual dependency is combined with intense social pressures, we might expect the computation of eye movements to have a more abstract and generalized component than limb or hand movements. Since, in the macaque, one portion of BA 45 is closely linked to eye movements, while the other makes long-distance cortical connections, I suggest that the abstract and amodal computations involved in language (whether spoken or signed) had a pre-adaptive foundation in the social and visually guided aspects of gaze that are, by hypothesis, subserved by BA 45B.

These two hypotheses may in fact be complementary, in the sense that the special role of BA 44 in language production and processing may represent a kind of fusion of the two flanking regions, specifically the hierarchical premotor functions of BA 6, and the multi-modal, integrative, and social functions of BA 45. The result would be a more abstract computational process than hierarchical motor planning: an operator that can combine or unify pre-existing conceptual units (motor actions, vocalizations, or visual objects) to freely create a discrete infinity of modalityindependent cognitive structures. Such an operator shares the key functionality required by modern theories of language, such as 
Merge in minimalist approaches (Chomsky, 1995; Hendrick, 2003; Berwick and Chomsky, 2011) or "unify" in tree-adjoining, construction grammar or other unification grammars (cf. Hagoort, 2005b). Whether during comprehension or in production, such an operator must quickly retrieve items from memory (e.g., retrieve the phonological form of words from the lexicon), combine them in a context-relevant fashion (e.g., using background information and current context) into flexible, temporary, goal-relevant structures that can be parsed semantically (in comprehension) or produced motorically via some serialization process (during production). As emphasized by Hagoort, such an overarching computation is consistent with both the neuroanatomy of Broca's area, as discussed above, and a wide variety of brain imaging results focused on language comprehension (Hagoort, 2005a,b).

Could hierarchy-building circuitry in BA 6 and the deep frontal operculum, evolved in the context of motor planning and dedicated to motor control, be exapted to produce a general purpose, amodal, two-way circuit that can perform the computational equivalent of Merge or Unify? While this hypothesis clearly remains speculative at present, it has several points to recommend it:

1. We know from the brain imaging studies cited above that amodal, language circuitry exists in Broca's region and is involved in language production, regardless of output modality;

2. The modality-general portion of this circuitry appears to center on BA 45;

3. We know from both lesion and brain imaging studies that Broca's area more generally (BA 44 included) is involved not just in production by also in perception;

4. Studies of macaque mirror neurons and the human mirror system provide a plausible foundation for the two-way nature of this system, building on a pre-existing mirror system for interpreting the actions of others;

5. We know that Broca's area, broadly construed, can be activated in non-linguistic tasks, consistent with a generalized, amodal role of the LIFG in selection of appropriate action or items, and suppression of irrelevant alternatives.

Not only is this exaptive hypothesis consistent with the data above, but it makes several specific testable predictions about the structure, connectivity, and function of BA 45:

1. BA 45 in humans should be more amodal than other components of the LIFG, and in particular its white-matter connections should be longer, and fan out more widely, than those of other regions;

2. Anatomically, the cytoarchitecture of BA 45 should be less motor-driven, more perceptually embedded, and thus more suited to amodal cognition than BA 6 or BA 44 (as already suggested by its granular layer 4);

3. In monkeys, cells in BA 45A should fire in a much wider variety of situations than BA 44 or BA 6 (including in particular social cognition tasks).

I conclude that exaptive hypotheses about the origins of syntax, far from being "utterly implausible," are consistent with a wide range of neuroanatomical and functional data. I see such hypotheses, when built upon a solid foundation of comparative neuroscientific data, as presenting numerous testable predictions and avenues for profitable empirical investigation. Of course, "plausible" plus "testable" does not equal "true." But I hope to have convinced the open-minded reader that there are good reasons for considering exaptive hypotheses in research on the evolution of cognition, and that the mistrust or outright hostility such hypotheses have evoked from some quarters is unjustified.

\section{TESTING EXAPTIVE HYPOTHESES: THE ROLE OF COMPARATIVE RESEARCH}

I will end with a brief attempt to clarify the role of comparative research in testing evolutionary hypotheses in general, and exaptive hypotheses in particular. In brief, we can use comparison of homologous traits in closely related species to derive inferences about ancestral states. This is an important first step in testing any exaptive hypothesis: we must first understand the ancestral state that served as a putative "pre-adaptation." When considering the evolution of language, and neural components in particular, the comparison set here will typically be non-human primates, or sometimes mammals more generally.

The second set of comparisons involve "analogies" - similar traits that have evolved independently, via convergent evolution (such traits are one member of the broader class termed "homoplasies" by comparative biologists). Convergent evolution plays a central role in testing evolutionary hypotheses, because each example of a convergently evolved trait represents an independent evolutionary event, and thus an independent data point for statistical testing (Harvey and Pagel, 1991; Pagel, 1992). In contrast, a trait that is homologous among a group of species has, by definition, evolved only once in that clade, and even if there are hundreds of species sharing the trait, they constitute only a single independent data point.

The clade used most frequently in comparative tests regarding language evolution are birds, Class Aves, which have evolved numerous traits convergently with some mammals (e.g., homeothermy or "warm bloodedness," or large brains) and specifically with humans (e.g., biparental care, vocal imitation, or bipedalism).

\section{HOMOLOGY: INFERENCES ABOUT ANCESTRAL STATE}

A homologous trait is one that is shared among a group of related organisms by virtue of its presence in their common ancestor. When attempting to build a phylogeny, systematists distinguish between two classes of homology. "Synapomorphies" are traits that are shared in a particular clade, but lacking in other close relatives, while "symplesiomorphies" are more broadly shared (due to their present in a more ancient shared ancestor). Feathers are a synapomorphy among living bird species (all birds have them, and all living non-birds lack them), while the possession of a heart or a mouth are symplesiomorphies (all birds have them, but so do all other vertebrates). However, in the current context we are concerned with rebuilding ancestral states, however far back they might go, and so we will discuss homology in general.

Homology is a relative concept: it depends on what trait is being examined, and what particular clade is being discussed. Thus, for 
example, the wings of birds and bats are homologous as forelimbs (because they both derive from the forelimb of the shared tetrapod ancestor of birds and mammals) but are convergently evolved analogs as wings. Furthermore, correct determination of homology depends upon the level of mechanistic detail being discussed. The complex, image-forming eyes found in insects, octopus, or vertebrates evolved convergently (Allman, 1999) but their location is nonetheless controlled by a homologous transcription factor Pax-6 (Quiring et al., 1994; Gehring and Ikeo, 1999; van Heyningen and Williamson, 2002). Such a situation has been termed deep homology and appears to play a surprisingly important role in human evolution (cf. Carroll, 2008; Fitch, 2009; Shubin et al., 2009).

Careful examination of homologous traits in multiple species allows us to reconstruct traits that were present in the common ancestor of those species. For example, the corpus callosum is found in all placental mammals (eutherians), but is absent in marsupials and monotremes. This allows us to conclude that the corpus callosum was not present in the common ancestor of all mammals, but arose in the LCA of living eutherians. In contrast, the anterior commissure is found in marsupials, and more widely among vertebrates including birds, suggesting that it evolved rather early in tetrapods. Such inferences about ancestral states depend on solid comparative neuroanatomy in living organisms, and no fossil evidence is required to roughly date such evolutionary events.

A broad comparative analysis is also important to determine the directionality of any evolutionary changes in different lineages. It is important to note that traits can be lost as well as gained in evolution. There is no way to know, based on a simple comparison of two species that differ in some trait, what the directionality of change might have been, and this requires outgroup comparisons with other related species. For example, one might think that the sexual swellings surrounding the vaginal area in female chimpanzees are a primitive feature of primates, given that such swellings are also seen in macaques and baboons. This would lead to the conclusion that humans have lost such swellings in our recent evolutionary history. An outgroup comparison, looking at gorillas, orangutans, and gibbons (the other members of our own ape clade) refutes that intuitive inference: none of these apes shows sexual swellings. Thus in this case humans retain the primitive state, and it is chimpanzees that have evolved a novelty (convergently with moneys and baboons). This example illustrates both the need for rigorous outgroup comparisons when analyzing homology, and also illustrates that the LCA of humans and chimpanzees was not a chimpanzee. In this case, and presumably many others, chimpanzees have evolved, just as we have, in the last 6 million years.

\section{CONVERGENCE: TESTING HYPOTHESES ABOUT MECHANISM AND ADAPTIVE VALUE}

Homologies play a crucial role in constructing phylogenies and rebuilding extinct ancestors. For such problems, convergent evolution is a nuisance and a distraction. But for a different set of more abstract evolutionary problems, convergent evolution provides one of the most powerful tools at our disposal, and thus the second main arm of the comparative method. These are problems involving statistically valid tests of evolutionary hypotheses, whether of mechanism or evolutionary function.

In some cases convergent evolution of a similar trait can lead us to new hypotheses about function. Consider the example of the descended human larynx, which was until recently believed to be uniquely human, and therefore assumed to be directly tied to human speech abilities. The recent discoveries of a permanently descended larynx in several deer species, big cats such as lions and tigers, koalas, Mongolian gazelles, and goitered gazelles clearly demonstrates that a descended larynx can serve some purpose other than speech (Fitch, 2010). In this case, analyses strongly suggest that the purpose is an exaggeration of the size conveyed by vocalizations, so that animals with a descended larynx seem larger to listeners. Because this explanation also applies to humans, it offers a clear alternative to the standard idea that the descended larynx is an exclusively speech-related trait. This is particularly true since the human larynx descends a second time, at puberty, but only in males. This sexually dimorphic descent seems unlikely to be tied to speech abilities, since teenage males do not undergo any improvement in these: instead it is simply part of a deeper, richer, and more impressive voice timbre, quite similar to the red deer's roar.

In other cases convergent evolution of mechanism can corroborate mechanistic hypotheses. A nice example is given by the evolution of direct motor connections in birds. As discussed above in hypothesis one, a plausible reason chimpanzees and other primates cannot talk, and humans can, is because humans possess direct cortico-motor connections for laryngeal control, and other primates do not. This is a correlational argument: humans have speech and have direct connections, and other primates do not. Therefore, direct connections are involved in speech motor control. While plausible, such arguments are never particularly convincing. However, in this case, data from birds strongly support the argument, because direct connections from the avian equivalent of motor cortex (area RA) indeed have direct monosynaptic connections onto the motor neurons controlling the muscles of the syrinx (the birds primary organ of voice). These connections are lacking in bird species incapable of vocal learning (Wild, 1993a,b). This case, where an analogous mechanism is present in species which have convergently evolved the analogous ability, lends great credence to the Kuypers/Jürgens hypothesis. What is more, there are many other relevant species in which to test this prediction, including all the mammals who have convergently evolved vocal learning.

Many other examples of the power of convergent evolution could be drawn from the genetic level, already explored in previous work. For example, the role of FOXP2 in vocal control and vocal motor learning has been elegantly explored in birds by Constance Scharff and colleagues (Haesler et al., 2004, 2007; Scharff and Haesler, 2005), and provides the first example of deep homology yet known in the domain of vocal motor control (cf. Fitch, 2009; Scharff and Petri, 2011). Research on axon guidance has examined the development of a set of connections in the avian brain that may be the analogy of the arcuate fasciculus, and suggests that a set of broadly shared axonal guidance molecules, the cadherins, may play a key role in this (Matsunaga and Okanoya, 2008, 2009). An intriguing possibility is that these same molecules, or close relatives, might also play a role in the development of 
intracortical connections that underlie syntax or spoken language more broadly.

In summary then, both analogy and homology will always have a role to play in the comparative method, and the importance of convergent evolution for testing evolutionary hypotheses means that research on the avian brain will continue to be extremely important in analyses of traits that make humans unique among primates.

\section{CONCLUSION}

In this paper I have shown that models of neural/cognitive evolution that invoke exaptation - changes in function - are fully consistent with Darwin's ideas about evolution by natural selection, as well as with modern evolutionary biology and contemporary neuroscience. To illustrate this point I have assembled three exaptive hypotheses, focused on different mechanisms of human spoken

\section{REFERENCES}

Aitken, P. G. (1981). Cortical control of conditioned and spontaneous vocal behavior in rhesus monkeys. Brain Lang. 13, 171-184.

Allman, J. M. (1999). Evolving Brains. (New York: Scientifc American Library).

Allott, R. M. (1994). "Motor theory of language origin: the diversity of languages," in Studies in Language Origins, eds J. Wind, A. Jonker, R. M. Allott, and L. Rolfe (Amsterdam: John Benjamins), 125-160.

Amunts, K., Schleicher, A., Bürgel, U., Mohlberg, H., Uylings, H. B. M., and Zilles, K. (1999). Broca's region revisited: cytoarchitecture and intersubject variability. J. Comp. Neurol. 412, 319-341.

Anwander, A., Tittgemeyer, M., von Cramon, D. Y., Friederici, A. D., and Knos̈che, T. R. (2007). Connectivitybased parcellation of Broca's area. Cereb. Cortex 17, 816-825.

Arbib, M. A. (2005). From monkey-like action recognition to human language: an evolutionary framework for neurolinguistics. Behav. Brain Sci. 28, 105-167.

Bahlmann, J., Schubotz, R. I., and Friederici, A. D. (2008). Hierarchical artificial grammar processing engages Broca's area. Neuroimage 42, 525-534.

Bellugi, U., Poizner, H., and Klima, E. S. (1990). "Mapping brain funcion for language: evidence for sign language," in Signal and Sense, eds G. M. Edelman, W. E. Gall, and W. M. Cowan (New York, NY: John Wiley \& Sons, Inc.), 521-543.

Berwick, R. C. (1997). Syntax facit saltum: computation and the genotype and phenotype of language. $J$. Neurolinguistics 10, 231-249.

Berwick, R. C., and Chomsky, N. (2011). "The biolinguistic program: the current state of its development," in The
Biolinguistic Enterprise: New Perspectives on the Evolution and Nature of the Human Language Faculty, eds A. M. Di Sciullo and C. Boeckx (Oxford: Oxford University Press), 19-41.

Bickerton, D. (1990). Language and Species. Chicago, IL: Chicago University Press.

Bickerton, D. (2000). "How protolanguage became language," in The Evolutionary Emergence of Language: Social Function and the Origins of Linguistic Form, eds C. Knight, M. Studdert-Kennedy, and J. R. Hurford (Cambridge: Cambridge University Press), 264-284.

Bickerton, D. (2007). Language evolution: a brief guide for linguists. Lingua 117, 510-526.

Bickerton, D. (2010). Adam's Tongue, New York: Hill and Wang.

Brown, S. (2000). "The "Musilanguage" model of music evolution," in The Origins of Music, eds N. L. Wallin, B. Merker, and S. Brown (Cambridge, MA: The MIT Press), 271-300.

Buss, D. M., Haselton, M. G., Shackelford, T. K., Bleske, A. L., and Wakefield, J. C. (1998). Adaptations, exaptations, and spandrels. Am. Psychol. 53, 533-548.

Byrne, R. (1997). Machiavellian intelligence. Evol. Anthropol. 5, 172-180.

Caramazza, A., and Zurif, E. B. (1976). Dissociation of algorithmic and heuristic processes in language comprehension: evidence from aphasia. Brain Lang. 3, 572-582.

Carroll, S. B. (2008). Evo-devo and an expanding evolutionary synthesis: a genetic theory of morphological evolution. Cell 134, 25-36.

Catani, M., and Mesulam, M. (2008). The arcuate fasciculus and the disconnection theme in language and aphasia: history and current state. Cortex 44, 953-961. language, to try to illustrate this approach from a "nuts and bolts" perspective, and to make some specific predictions that could be tested. Although I find each of these hypotheses plausible, I have no personal investment in them being true in any ultimate sense. But I do believe that they have a virtue that all good scientific hypotheses share: they are testable. Specific, testable hypotheses remain all too rare in research on the evolution of language. Furthermore, such hypotheses present numerous avenues for testing and elaboration using the comparative method, both for pinpointing ancestral states via homology, and for testing hypotheses using convergence.

Since testing plausible hypotheses is the surest route to progress I know of in science, I would be very pleased to see any of the three hypotheses above tested, even if refuted in the process. If that occurs, a new and better hypothesis will surely rise to take its place.

Catania, K. C. (1999). A nose that looks like a hand and acts like an eye: the unusual mechanosensory system of the star-nosed mole. J. Comp. Physiol. A 185, 367-372.

Chomsky, N. (1968). Language and Mind. New York: Harcourt, Brace \& World.

Chomsky, N. (1976). On the nature of language. Ann. N. Y. Acad. Sci. 280, 46-57.

Chomsky, N. (1995). The Minimalist Program. Cambridge, MA: MIT Press.

Chomsky, N. (2010). "Some simple evo devo theses: how true might they be for language?" in The Evolution of Human Language: Biolinguistic Perspectives, eds R. Larson, V. Deprez, and H. Yamakido (Cambridge: Cambridge University Press), 45-62.

Clark, B. (2011). Scavening, the stag hun, and the evolution of language. J. Linguist. 47, 447-480.

Darwin, C. (1871). The Descent of Man and Selection in Relation to Sex. London: John Murray.

Deacon, T. W. (1992). "The neural circuitry underlying primate calls and human language," in Language Origins: A Multidisciplinary Approach, eds J. Wind, B. A. Chiarelli, B. Bichakjian, and A. Nocentini (Dordrecht: Kluwer Academic), 301-323.

Deacon, T. W. (1997). The Symbolic Species: The Co-Evolution of Language and the Brain. New York: Norton.

Dennett, D. C. (1995). Darwin's Dangerous Idea. New York: Simon \& Schuster.

di Pellegrino, G., Fadiga, L., Fogassi, L. Gallese, V., and Rizzolatti, G. (1992). Understanding motor events: a neurophysiological study. Exp. Brain Res. 91, 176-180.

Emery, N. J. (2000). The eyes have it: the neuroethology, function and evolution of social gaze. Neurosci. Biobehav. Rev. 24, 581-604.

Emery, N. J., Lorincz, E. N., Perrett, D. I., Oram, M. W., and Baker, C. I. (1997). Gaze following and joint attention in rhesus monkeys (Macaca mulatta). J. Comp. Psychol. 111, 286-293.

Feenders, G., Liedvogel, M., Rivas, M., Zapka, M., Horita, H., Hara, E., Wada, K., Mouritsen, H., and Jarvis, E. D. (2008). Molecular mapping of movement-associated areas in the avian brain: a motor theory for vocal learning origin. PLoS ONE 3, e1768. doi:10.1371/journal.pone.0001768

Fitch, W. T. (2002). "Comparative vocal production and the evolution of speech: reinterpreting the descent of the larynx," in The Transition to Language, ed. A. Wray (Oxford: Oxford University Press), 21-45.

Fitch, W. T. (2006). The biology and evolution of music: a comparative perspective. Cognition 100, 173-215.

Fitch, W. T. (2009). "The biology \& evolution of language: "deep homology" and the evolution of innovation," in The Cognitive Neurosciences IV, ed. M. S. Gazzaniga (Cambridge, MA: MIT Press), 873-883.

Fitch, W. T. (2010). The Evolution of Language. Cambridge: Cambridge University Press.

Fitch, W. T., Huber, L., and Bugnyar, T. (2010). Social cognition and the evolution of language: constructing cognitive phylogenies. Neuron 65, 795-814.

Fitch, W. T., and Reby, D. (2001). The descended larynx is not uniquely human. Proc. Biol. Sci. 268, 1669-1675.

Foerster, O. (1936). "Motorische Felder und Bahnen," in Handbuch der Neurologie, eds Band IV, O. Bumke, and O. Foerster (Berlin: Springer), $1-448$. 
Frey, R., and Riede, T. (2003). Sexual dimorphism of the larynx of the Mongolian Gazelle (Procapra gutturosa Pallas, 1777) (Mammalia, Artiodactyla, Bovidae). Zool. Anz. 242, 33-62.

Friederici, A., Meyer, M., and von Cramon, D. Y. (2000). Auditory language comprehension: an eventrelated fMRI study on the processing of syntactic and lexical information. Brain Lang. 74, 289-300.

Friederici, A. D., Bahlmann, J., Heim, S., Schubotz, R. I., and Anwander, A. (2006). The brain differentiates human and non-human grammars: functional localization and structural connectivity. Proc. Natl. Acad. Sci. U.S.A. 103, 2458-2463.

Galantucci, B., Fowler, C. A., and Turvey, M. T. (2006). The motor theory of speech perception reviewed. Psychon. Bull. Rev. 13, 361-377.

Gehring, W. J., and Ikeo, K. (1999). Pax 6: mastering eye morphogenesis and eye evolution. Trends Genet. $15,371-377$.

Gerbella, M., Belmalih, A., Borra, E., Rozzi, S., and Luppino, G. (2010). Cortical connections of the macaque caudal ventrolateral prefrontal areas 45A and 45B. Cereb. Cortex 20, 141-168.

Geschwind, N. (1965). Disconnexion syndromes in animals and man. Brain 88, 585-644.

Geschwind, N. (1970). The organization of language and the brain. Science $170,940-944$.

Gould, S. J. (1991). Exaptation: a crucial tool for evolutionary psychology. $J$. Soc. Issues 47, 43-65.

Gould, S. J., and Vrba, E. S. (1982). Exaptation-a missing term in the science of form. Paleobiology 8, 4-15.

Grossman, M. (1980). A central processor for hierarchically-structured material: evidence from Broca's aphasia. Neuropsychologia 18, 299-308.

Groswasser, Z., Korn, C., GroswasserReider, J., and Solzi, P. (1988). Mutism associated with buccofacial apraxia and bihemispheric lesions. Brain Lang. 34, 157-168.

Haesler, S., Rochefort, C., Geogi, B., Licznerski, P., Osten, P., and Scharff, C. (2007). Incomplete and inaccurate vocal imitation after knockdown of FoxP2 in songbird basal ganglia nucleus area $\mathrm{X}$. PLoS Biol. 5, e321. doi:10.1371/journal.pbio. 0050321

Haesler, S., Wada, K., Nshdejan, A., Morrisey, E. E., Lints, T., Jarvis, E. D., and Scharff, C. (2004). FoxP2 expression in avian vocal learners and non-learners. J. Neurosci. 24, 3164-3175.
Hagoort, P. (2005a). "Broca’s complex as the unification space for language," in Twenty-First Century Psycholinguistics: Four Cornerstones, ed. A. Cutler (London: Lawrence Erlbaum), 157-172.

Hagoort, P. (2005b). On Broca, brain, and binding: a new framework. Trends Cogn. Sci. 9, 416-423.

Harvey, P. H., and Pagel, M. D. (1991). The Comparative Method in Evolutionary Biology. Oxford: Oxford University Press.

Hayes, C. (1951). The Ape in Our House. New York: Harper.

Hendrick, R. (ed.). (2003). Minimalist Syntax. Oxford: Blackwell.

Hewes, G. W. (1973). Primate communication and the gestural origin of language. Curr. Anthropol. 14 5-24.

Holstege, G., and Ehling, T. (1996). "Two motor systems involved in the production of speech," in Vocal Fold Physiology: Controlling Complexity and Chaos, eds P. J. Davis and N. H. Fletcher (San Diego: Singular Press), 153-196.

Horwitz, B., Amunts, K., Bhattacharyya, R., Patkin, D., Jeffries, K., Zilles, K., and Braun, A. R. (2003). Activation of Broca's area during the production of spoken and signed language: a combined cytoarchitectonic mapping and PET analysis. Neuropsychologia 41, 1868-1876.

Humphrey, N. K. (1976). "The social function of intellect," in Growing Points in Ethology, eds P. P. G. Bateson and R. A. Hinde (Cambridge: Cambridge University Press), 303-317.

Iacoboni, M., Molnar-Szakacs, I., Gallese, V., Buccino, G., Mazziotta, J. C., and Rizzolatti, G. (2005). Grasping the intentions of others with one's own mirror neuron system. PLoS Biol. 3, e79. doi:10.1371/journal.pbio.0030079

Jackendoff, R., and Pinker, S. (2005). The nature of the language faculty and its implications for evolution of language (Reply to Fitch, Hauser, \& Chomsky). Cognition 97, 211-225.

Jacob, F. (1977). Evolution and tinkering. Science 196, 1161-1166.

Jacob, F. (1982). The Possible and the Actual. New York: Pantheon.

Jolly, A. (1966). Lemur social behavior and primate intelligence. Science $153,501-506$.

Jürgens, U. (1994). The role of the periaqueductal grey in vocal behaviour. Behav. Brain Res. 62, 107-117.

Jürgens, U., Kirzinger, A., and von Cramon, D. Y. (1982). The effects of deep-reaching lesions in the cortical face area on phonation: a combined case report and experimental monkey study. Cortex 18, 125-139.

Keysers, C., and Gazzola, V. (2006). Towards a unifying neural theory of social cognition. Prog. Brain Res. 156, 379-401.

Kimura, D. (1993). Neuromotor Mechanisms in Human Communication. Oxford: Oxford University Press.

Kingsolver, J. G., and Koehl, M. A. R. (1985). Aerodynamics, thermoregulation, and the evolution of insect wings: differential scaling and evolutionary change. Evolution 39, 488-504.

Kuypers, H. G. J. M. (1958a). Corticobulbar connections to the pons and lower brainstem in man: an anatomical study. Brain 81 364-388.

Kuypers, H. G. J. M. (1958b). Some projections from the pericentral cortex to the pons and lower brain stem in monkey and chimpanzee. J. Comp. Neurol. 110, 221-255.

Kuypers, H. G. J. M. (1973). "The anatomical organization of the descending pathways and their contributions to motor control especially in primates," in New Developments in EMG and Clinical Neurophysiology, ed. J. E. Desmedt (Basel: Karger), 38-68.

Lashley, K. (1951). “The problem of serial order in behavior," in Cerebral Mechanisms in Behavior; The Hixon Symposium, ed. L. A. Jeffress (New York: Wiley), 112-146.

Liberman, A. M., Cooper, F. S. Shankweiler, D. P., and StuddertKennedy, M. (1967). Perception of the speech code. Psychol. Rev. 74 431-461.

Liberman, A. M., and Mattingly, I. G. (1985). The motor theory of speech perception revised. Cognition 21 $1-36$.

Lieberman, P. (1968). Primate vocalization and human linguistic ability. $J$. Acoust. Soc. Am. 44, 1574-1584.

Lieberman, P. (1975). On the Origins of Language. New York: Macmillan.

Lieberman, P. (1984). The Biology and Evolution of Language. Cambridge, MA: Harvard University Press.

Lieberman, P. (2000). Human Language and Our Reptilian Brain: the Subcortical Bases of Speech, Syntax and Thought. Cambridge, MA: Harvard University Press.

MacNeilage, P. F. (1998). The frame/content theory of evolution of speech production. Behav. Brain Sci. 21, 499-546.

Marshall, A. J., Wrangham, R. W., and Arcadi, A. C. (1999). Does learning affect the structure of vocalizations in chimpanzees? Anim. Behav. 58, 825-830.

Matsunaga, E., and Okanoya, K. (2008). Expression analysis of cadherins in the songbird brain: relationship to vocal system development. J. Comp. Neurol. 508, 329-342.

Matsunaga, E., and Okanoya, K. (2009). Evolution and diversity in avian vocal system: an Evo-Devo model from the morphological and behavioral perspectives. Dev. Growth Differ. 51, 355-367.

Mithen, S. (2005). The Singing Neanderthals: The Origins of Music, Language, Mind, and Body. London: Weidenfeld \& Nicolson.

Myers, R. E. (1976). Comparative neurology of vocalization and speech: proof of a dichotomy. Ann. N. Y. Acad. Sci. 280, 745-757.

Newmeyer, F. J. (1998). "On the supposed 'counterfunctionality' of universal grammar: some evolutionary implications," in Approaches to the Evolution of Language, eds J. R. Hurford, M. Studdert-Kennedy, and C. Knight (New York: Cambridge University Press), 305-319.

Nimchinsky, E. A., Gilissen, E., Allman, J. M., Perl, D. P., Erwin, J. M., and Hof, P. R. (1999). A neuronal morphologic type unique to humans and great apes. Proc. Natl. Acad. Sci. U.S.A. 96, 5268-5273

Pagel, M. D. (1992). A method for the analysis of comparative data. $J$. Theor. Biol. 156, 434-442.

Piattelli-Palmarini, M. (1994). Ever since language and learning: afterthoughts on the PiagetChomsky debate. Cognition 50, 315-346.

Pinker, S., and Bloom, P. (1990). Natural language and natural selection. Behav. Brain Sci. 13, 707-784.

Pinker, S., and Jackendoff, R. (2005). The faculty of language: what's special about it? Cognition 95, 201-236.

Pulvermüller, F., and Fadiga, L. (2010). Active perception: sensorimotor circuits as a cortical basis for language. Nat. Rev. Neurosci. 11, 351-360.

Quiring, R., Walldorf, U., Kloter, U., and Gehring, W. J. (1994). Homology of the eyeless gene of Drosophila to the small eye gene in mice and aniridia in humans. Science 265, 785-789.

Reeve, H. K., and Sherman, P. (1993). Adaptation and the goals of evolutionary research. Q. Rev. Biol. 68, $1-32$.

Rempel-Clower, N. L., and Barbas, H. (2000). The laminar pattern of connections between prefrontal and anterior temporal cortices in the rhesus monkey is related to cortical structure and function. Cereb. Cortex 10, 851-865. 
Reynolds Losin, E. A., Russell, J. L., Freeman, H., Meguerditchian, A., and Hopkins, W. D. (2008). Left hemisphere specialization for oro-facial movements of learned vocal signals by captive chimpanzees. PLoS ONE 3, e2529. doi:10.1371/journal.pone.0002529

Richman, B. (1993). On the evolution of speech: singing as the middle term. Curr. Anthropol. 34, 721-722.

Rilling, J. K., Glasser, M. F., Preuss, T. M., Ma, X., Zhao, T., Hu, X., and Behrens, T. E. J. (2008). The evolution of the arcuate fasciculus revealed with comparative DTI. Nat. Neurosci. 11 4, 426-428.

Rilling, J. K., and Seligman, R. A. (2002). A quantitative morphometric comparative analysis of the primate temporal lobe. J. Hum. Evol. 42, 505-533.

Roe, A. W., Pallas, S. L., Kwon, Y. H., and Sur, M. (1992). Visual projection routed to the auditory pathway in ferrets: receptive fields of visual neurons in primary auditory cortex. J. Neurosci. 12, 3651-3664.

Scaife, M., and Bruner, J. S. (1975). The capacity for joint visual attention in the infant. Nature 253, 265-266.
Scharff, C., and Haesler, S. (2005). An evolutionary perspective on FoxP2: strictly for the birds? Curr. Opin. Neurobiol. 15, 694-703.

Scharff, C., and Petri, J. (2011). Evodevo, deep homology and FoxP2: implications for the evolution of speech and language. Philos. Trans. R. Soc. Lond. B 366, 2124-2140.

Schoenemann, P. T., Sheehan, M. J. and Glotzer, L. D. (2005). Prefrontal white matter volume is disproportionately larger in humans than in other primates. Nat. Neurosci. 8, 242-253.

Shubin, N., Tabin, C., and Carroll, S. (2009). Deep homology and the origins of evolutionary novelty. Nature $457,818-823$.

Striedter, G. F. (2004). Principles of Brain Evolution. Sunderland, MA: Sinauer.

Suga, N., Niwa, H., Taniguchi, I., and Margoliash, D. (1987). The personalized auditory cortex of the mustached bat: adaptation for echolocation. J. Neurophysiol. 58, 643-654.

Sutton, D., Larson, C., and Lindeman, R. C. (1974). Neocortical and limbic lesion effects on primate phonation. Brain Res. 71, 61-75.

van Heyningen, V., and Williamson, K. A. (2002). PAX6 in sensory development. Hum. Mol. Genet. 11, 1161-1167.

Weissengruber, G. E., Forstenpointner, G., Peters, G., Kübber-Heiss, A., and Fitch, W. T. (2002). Hyoid apparatus and pharynx in the lion (Panthera leo), jaguar (Panthera onca), tiger (Panthera tigris), cheetah (Acinonyx jubatus), and domestic cat (Felis silvestris f. catus). J. Anat. 201, 195-209.

Wernicke, C. (1874). Der Aphasische Symptomenkomplex. Breslau: Cohn and Weigert.

Wild, J. M. (1993a). The avian nucleus retroambigualis: a nucleus for breathing, singing and calling. Brain Res. 606, 119-124.

Wild, J. M. (1993b). Descending projections of the songbird nucleus robustus archistriatalis. J. Comp. Neurol. 338, 225-241.

Yerkes, R. M., and Yerkes, A. W. (1929). The Great Apes. New Haven, CT: Yale University Press.
Zilles, K. (2004). "Architecture of the human cerebral cortex: regional and laminar organization," in The Human Nervous System, eds G. Paxinos and J. K. Mai (Amsterdam: Elsevier), 997-1055.

Conflict of Interest Statement: The author declares that the research was conducted in the absence of any commercial or financial relationships that could be construed as a potential conflict of interest.

Received: 03 November 2011; accepted: 02 December 2011; published online: 23 December 2011.

Citation: Fitch WT (2011) The evolution of syntax: an exaptationist perspective. Front. Evol. Neurosci. 3:9. doi: 10.3389/fnevo.2011.00009

Copyright (c) 2011 Fitch. This is an open-access article distributed under the terms of the Creative Commons Attribution Non Commercial License, which permits non-commercial use, distribution, and reproduction in other forums, provided the original authors and source are credited. 